\title{
AVALIAÇÃO DAS PRESCRIÇÕES AVIADAS NA UNIDADE DE PRONTO ATENDIMENTO NO MUNICÍPIO DE IJUÍ/RS
}

\section{Evaluation of prescriptions dispensed in the emergency care unit in the city of Ijuí/RS}

\author{
Aline SCHNEIDER ${ }^{1}$ \\ Gislaine DAL MOLIN ${ }^{2}$ \\ Marilei Uecker PLETSCH ${ }^{3}$ \\ Maristela CODINOTTI ${ }^{4}$ \\ Christiane de Fátima COLET ${ }^{3}$
}

Recebido em: 22/05/2014 - Aprovado em: 12/09/2014 - Disponibilizado em: 15/12/2014

\begin{abstract}
Resumo
O objetivo do estudo foi avaliar aspectos relativos a prescrição de medicamentos em prescrições aviadas na Farmácia da Unidade de Pronto Atendimento no município de Ijuí/RS, durante o mês de julho de 2012. Foram avaliadas, de forma retrospectiva, as segundas vias das prescrições que ficam retidas no local de estudo, havendo dupla conferência da legibilidade das mesmas. Foi utilizada a Relação de Medicamentos Essenciais do Município de Ijuí, vigente em 2012, sendo que os medicamentos prescritos em cada receita foram agrupados de acordo com a classificação ATC da OMS. As variáveis da pesquisa foram analisadas de forma descritiva e matemático-estatística, utilizando frequência absoluta, porcentagem, média e desvio padrão. Foram aviadas 1.138 prescrições, a faixa etária que mais prevaleceu foi de $0-9$ anos (24\%), seguido pela faixa etária de 20-29 anos (17\%). A maioria dos usuários era do sexo feminino (56\%). Os grupos de medicamentos mais prescritos foram os analgésicos $(22,5 \%)$, seguido de antibacterianos de uso sistêmico $(18,9 \%)$ e antiinflamatórios e anti-reumáticos $(17,6 \%)$. Os medicamentos mais prescritos, no primeiro nível da ATC, foram os que agem sobre sistema nervoso. Pode-se observar que a maioria das prescrições continham medicamentos da REMUME. O número médio de medicamentos por receita foi maior do que o sugerido pela OMS. Essa observação, assim como os outros dados, pode direcionar esforços gerenciais e educativos no sentido da promoção do uso racional de medicamentos e atingir resultados mais próximos dos preconizados pelos órgãos internacionais.
\end{abstract}

Palavras-chave: Medicamentos, Prescrição, Uso racional de medicamentos.

\begin{abstract}
The objective study was to evaluate aspects related to prescription drugs, in the city of Ijuí / RS, with prescriptions dispensed at the Pharmacy Emergency Unit, during the month of July 2012. Were evaluated, retrospectively, the duplicates prescriptions that are retained in the study site, with double checking the readability of the same. We used the ratio of Essential Medicines Municipality Ijuí force in 2012, and the medicines prescribed for each recipe were grouped according to the WHO ATC classification. The research variables were analyzed descriptively and mathematicalstatistical, using absolute frequency, percentage, mean and standard deviation . 1,138 prescriptions were dispensed, the most prevalent age group was 0-9 years (24\%), followed by the age group of 20-20 years (17\%). Most users were female $(56 \%)$. The groups most frequently prescribed drugs were analgesics $(22.5 \%)$, followed by antibiotics for systemic use (18.9\%) and Anti-inflammatory and antirheumatic products (17.6\%). The most frequently prescribed medications in the first level of the ATC were the effect on the nervous system. It can be observed that most of the prescriptions of medicaments REMUME. The mean number of drugs per prescription was greater than that suggested by WHO. This observation, as well as other data, can direct managerial and educational efforts towards the promotion of rational use of medicines and achieve results closer to those recommended by international organizations.

\footnotetext{
${ }^{1}$ Acadêmica do Curso de Farmácia da UNIJUI - aline_schneider90@ hotmail.com

${ }^{2}$ Acadêmica do Curso de Farmácia da UNIJUI

${ }^{3}$ Professora Mestre do Departamento de Ciências da Vida da UNIJUÍ

${ }^{4}$ Farmacêutica Responsável pela Farmácia de Pronto Atendimento do município de Ijuí
} 


\section{INTRODUÇÃO}

O Sistema Único de Saúde (SUS) assegura o acesso aos medicamentos, mediante a garantia da execução integral da Assistência Farmacêutica, a qual tem sido objeto de importantes reflexões no processo de discussão e aprofundamento das diferentes questões relativas à saúde. A eficácia da prestação dos serviços de saúde exige a compreensão e o domínio de conhecimentos científicos, tecnológicos e gerenciais que atendam aos objetivos coletivos e individuais, bem como aos aspectos técnicos específicos e sociais aplicáveis, voltados para a promoção e recuperação da saúde (MARIN, et al.; 2003, ALBUQUERQUE e TAVARES; 2011).

Dentro das politicas do SUS a Política Nacional de Medicamentos afirma que a Assistência Farmacêutica contempla algumas prioridades, entre as quais se destacam a promoção do Uso Racional de Medicamentos (URM) (BRASIL; 1998). A prescrição médica é um instrumento essencial na terapêutica e para o URM, pois deve conter as informações necessárias sobre o medicamento: a dose, a frequência e a duração do tratamento adequado para o(s) problema(s) do paciente, estando diretamente relacionada com a qualidade da terapêutica medicamentosa (MARIN, et al.; 2003). Tratase, portanto, de um importante fator para a qualidade e quantidade do consumo de medicamentos, embora o ato da prescrição sofra influências do conhecimento do prescritor, das expectativas do paciente e da indústria farmacêutica (PORTELA, et al.; 2007, ALBUQUERQUE e TAVARES; 2011).

A prescrição de medicamentos e os problemas relacionados a ela são assuntos de grande complexidade, sendo assim, indicadores podem ser utilizados para avaliar a qualidade de uma prescrição. Os indicadores padronizados para pesquisas, sugeridos pela Organização Mundial da Saúde (OMS) (2005), facilitam a coleta de dados de maneira organizada e passível de comparação com outros serviços (OMS; 2005). Estes Indicadores são medidas qualitativas ou quantitativas que avaliam desempenhos, detectam problemas e orientam a solução. Eles incluem o número médio de medicamento por prescrição, a porcentagem de medicamentos prescritos pela denominação genérica, a porcentagem de prescrições com pelo menos um antibiótico, a porcentagem de prescrição com pelo menos um medicamento injetável e a porcentagem de medicamentos prescritos que fazem parte da Lista de Medicamentos Essenciais (OMS, 2005).

\section{Segundo FROHLICH e MENGUE} (2011), os indicadores propostos são entendidos como medidas de primeira linha; afinal, qualidade não pode ser medida apenas 
através de um indicador. A intenção é estimular outros questionamentos e guiar para uma ação subsequente. Além disso, como os indicadores são desenvolvidos como medidas estatísticas quantitativas é importante continuar na investigação deles, com o intuito de aperfeiçoá-los e validá-los.

Em um estudo de prescrições médicas com o objetivo de avaliar a qualidade e as condições predisponentes a erros de medicação nas prescrições advindas do SUS do município de Ouro Preto, aplicando os indicadores de qualidade das prescrições da OMS, EV et al. (2008) verificaram que a qualidade das prescrições não estava de acordo com os indicadores preconizados pela OMS, ressaltando a necessidade da implementação de estratégias que reforcem a necessidade de uma prescrição correta. A falta de informações completas nas prescrições médicas avaliadas corrobora a necessidade de educação continuada dos prescritores e adoção de medidas para a melhoria da qualidade das prescrições.

Em outro estudo, Silvério e Leite (2010) avaliou-se a qualidade de prescrições que chegam a farmácias comunitárias para a dispensação em Minas Gerais, verificou-se que as prescrições não forneciam todas as informações necessárias para uma utilização correta e segura dos medicamentos; não estando, em sua maioria, legíveis, apresentando alto percentual de nomes comerciais, com baixos índices de medicamentos constantes na Relação Nacional de Medicamentos Essenciais (RENAME) e grande número de antibióticos.

A literatura disponibiliza vários estudos avaliando a qualidade das prescrições, no entanto esses estudos são realizados em Unidades Básicas de Saúde (SANTOS \& NITRINI; 2004, GIROTTO \& SILVA; 2006, FARIAS et al.; 2007, PORTELA et al.; 2007, NETO et al.; 2011). Porém as Unidades de Pronto Atendimento também são responsáveis por prescrições de medicamentos. Estas unidades se diferenciam das demais devido a administração de alguns medicamentos no momento do atendimento, não sendo necessária a prescrição para seguimento do tratamento (MARQUES \& LIMA; 2007). Sendo que em Unidades de Pronto Atendimento não foram encontrados estudos brasileiros.

Com o objetivo de melhorar as práticas de uso de medicamentos, dentro do contexto da Assistência Farmacêutica, o presente estudo teve por objetivo foi avaliar aspectos relativos a prescrição de medicamentos em prescrições aviadas na Farmácia da Unidade de Pronto Atendimento no município de Ijuí/RS, durante o mês de julho de 2012, na qual se avaliou a qualidade das prescrições médicas segundo os Indicadores de Qualidade de Prescrição da Organização Mundial da Saúde (OMS, 2005). 


\section{MÉTODOS}

O presente trabalho foi desenvolvido no município de Ijuí/RS, com prescrições aviadas na Farmácia da Unidade de Pronto Atendimento, durante o mês de julho de 2012. Foram avaliadas, de forma retrospectiva, as segundas vias das prescrições que ficam retidas no local de estudo.

Os itens das prescrições foram classificados de acordo com Indicadores de Qualidade da Prescrição, preconizados pela OMS (2005). Para avaliar a legibilidade da prescrição considerou-se: a) ilegível - quando apresentou mais de três palavras de difícil leitura; b) pouco legível - quando apresentou um a três itens de difícil leitura e c) legível quando houve total entendimento da prescrição, quanto a letra. A avaliação da legibilidade da prescrição foi realizada por acadêmicos de farmácia, havendo dupla conferência das mesmas. Para fins de estudo, considerou-se polifarmácia o uso de cinco ou mais medicamentos por prescrição (CARVALHO et al.; 2012).

A porcentagem de medicamentos prescritos pelo nome genérico (\%MG) foi calculada pela fórmula, segundo a metodologia de Portela et al. (2012):

A porcentagem de medicamentos prescritos que figuram na lista de medicamentos padronizados (\%MP) foi obtida a partir do cálculo:

$\% \mathrm{MP}=$ (Número de medicamentos prescritos da $\mathrm{LMP}^{5}$ / Número total de medicamentos prescritos) $\mathrm{X} 100$

Foi utilizada a Relação de Medicamentos Essenciais do Município de Ijuí (REMUME), vigente em 2012, para identificação dos medicamentos padronizados, observando-se o princípio ativo, a concentração e forma farmacêutica, independente de apresentar-se com nome genérico ou nome comercial.

Os medicamentos prescritos em cada receita foram agrupados de acordo com a classificação ATC (Anatomical Therapeutic Chemical Classification), da Organização Mundial de Saúde, para os estudos de utilização de medicamentos (WHO, 2000). Para o cálculo da frequência de medicamentos pelo segundo nível da ATC, utilizou-se como denominador $\mathrm{o}$ total de medicamentos prescritos e não, o total de prescrições.

As variáveis da pesquisa foram analisadas de forma descritiva e matemáticoestatística, utilizando frequência absoluta, porcentagem, média e desvio padrão.

A pesquisa foi aprovada pelo Comitê de Ética em Pesquisa da Universidade Regional do Noroeste do Estado do Rio Grande do Sul - UNIJUI, sob parecer consubstanciado $\mathrm{n}^{\circ}$ 96.400/2012.

\footnotetext{
${ }^{5}$ LMP - Lista de Medicamentos Padronizados
} 


\section{RESULTADOS}

Foram aviadas 1.138 prescrições. A faixa etária dos usuários para os quais as prescrições se destinam prevaleceu foi de 0-9 anos (24\%), seguido pela faixa etária de 20-29 anos (17\%). A maioria dos usuários eram do sexo feminino $(56 \%)$.

As características das prescrições estão dispostas na Tabela 1.

Tabela 1: Características da prescrição aviadas no mês de julho de 2012 na farmácia da Unidade de Pronto Atendimento. $\mathrm{n}=1.138$

\begin{tabular}{|c|c|c|c|}
\hline & \multicolumn{3}{|c|}{$n(\%)$} \\
\hline & Sim & & Não \\
\hline \multicolumn{4}{|l|}{ PACIENTE } \\
\hline Nome do paciente & $1119(98 \%)$ & & $8(2 \%)$ \\
\hline \multicolumn{4}{|l|}{ PRESCRITOR } \\
\hline Nome & $902(79 \%)$ & & $236(21 \%)$ \\
\hline Carimbo & $902(79 \%)$ & & $236(21 \%)$ \\
\hline$N^{0}$ de Registro & $594(52 \%)$ & & $544(48 \%)$ \\
\hline Especialidade & $59(6 \%)$ & & $1070(94 \%)$ \\
\hline \multicolumn{4}{|l|}{ PRESCRIÇÃO } \\
\hline Data & $1122(99 \%)$ & & $16(1 \%)$ \\
\hline \multirow[t]{2}{*}{ Legibilidade } & Legivel & Pouco Legível & Ilegivel \\
\hline & $877(77 \%)$ & $191(17 \%)$ & $67(6 \%)$ \\
\hline
\end{tabular}

Tabela 2: Características da prescrição aviadas no mês de julho de 2012 na farmácia da Unidade de Pronto Atendimento quanto aos Indicadores de qualidade da OMS (2005). $\mathrm{n}=1.138$

\begin{tabular}{|c|c|}
\hline Indicadores & \\
\hline Número médio de medicamentos por prescrição - $\mathrm{x} \pm \mathrm{dp}$ & $2,64 \pm 1,02$ \\
\hline Prescrição de antibióticos - n (\%) & $557(49 \%)$ \\
\hline Prescrição de injetável - n (\%) & $18(1,6 \%)$ \\
\hline Prescrição pela REMUME - n (\%) & $717(63 \%)$ \\
\hline Prescrição pelo nome genérico - n $(\%)$ & $297(26 \%)$ \\
\hline Total de medicamentos & $3005(100 \%)$ \\
\hline
\end{tabular}


Tabela 3: Medicamentos por classe farmacológica, classificados de acordo com segundo nível de classificação ATC.

\begin{tabular}{lcc}
\hline $\mathbf{2}^{\mathbf{0}}$ Nível de Classificação da ATC & N & \% \\
\hline Analgésicos (N02) & 678 & 22,5 \\
Antibacterianos de uso sistêmico (J01) & 569 & 18,9 \\
Antiinflamatórios e anti-reumáticos (M01) & 528 & 17,6 \\
Anti-histamínicos para uso sistêmico (R06) & 300 & 9,9 \\
Preparados para uso nasal (R01) & 200 & 6,6 \\
Agentes para desordens funcionais gastrointestinais (A03) & 148 & 4,9 \\
Antiasmáticos (R03) & 133 & 4,5 \\
Preparações para tosse e resfriado (R05) & 120 & 3,9 \\
Outros* & 110 & 3,7 \\
Antiácidos, preparações para tratamento de úlceras pépticas e flatulência & 73 & 2,5 \\
(A02) & & 2,4 \\
Reposição hidroeletrolítica oral (A07) & 64 & 2,4 \\
Reposição hidroeletrolítica oral (A07) & 64 & 1,4 \\
Oftalmológicos (S01) & 44 & 1,2 \\
Corticosteróides para uso sistêmico (H02) & 38 & \\
\hline
\end{tabular}

*Foram agrupados em "outros" todos os subgrupos que apresentaram frequência menor que $1 \%$.

A Tabela 2 mostra os dados das prescrições avaliados conforme os indicadores de qualidade da OMS (2005).

A Tabela 3 mostra a distribuição dos medicamentos prescritos por classe farmacológica.

Os grupos de medicamentos mais prescritos foram os analgésicos (22,5\%), seguido de antibacterianos de uso sistêmico $(18,9 \%)$ e antiinflamatórios e anti-reumáticos $(17,6 \%)$. Os medicamentos mais prescritos classificados no primeiro nível da ATC foram os de efeito no sistema nervoso.

Os grupos que não contabilizaram mais que $1 \%$ dos medicamentos prescritos correspondem a fármacos com ação no sistema renina-angiotensina (C09), diuréticos
(C03), antibióticos para uso tópico (D06), corticosteroides em preparações para uso tópico (D07), hormônios sexuais e moduladores do sistema genital (G03), e antimicóticos para uso sistêmico (J02).

\section{DISCUSSÃO}

A prescrição é um documento legal, pelo qual se responsabilizam quem prescreve e dispensa o medicamento, e por isso envolve questões de âmbito legal, técnico e clínico, estando seus responsáveis sujeitos à legislação de controle e às ações de vigilância sanitária. Em nosso estudo $2 \%$ das prescrições não continham o nome do paciente e mesmo assim foram aviadas pelos funcionários da farmácia. $21 \%$ das prescrições não continham 
o nome do prescritor e nem o seu carimbo, impossibilitando a identificação do profissional e a verificação da veracidade das informações contidas na prescrição.

A data da prescrição é importante para verificar a validade da mesma de acordo com a legislação vigente no município, porém em nosso estudo $1 \%$ das prescrições foram aviadas sem conter este item, podendo representar prescrições vencidas que não poderiam ser aviadas.

Quanto a legibilidade das prescrições os resultados obtidos neste estudo foram melhores do que os encontrados por SILVÉRIO \& LEITE (2010) em um estudo avaliando a qualidade das prescrições aviadas em farmácias comunitárias em um município de Minas Gerais, no qual 368 (32\%) prescrições foram consideradas legíveis, 367 (32\%) foram consideradas pouco legíveis e 421 (36\%) ilegíveis.

A média de medicamentos por prescrição (MP) de 2,64 foi similar ao encontrado por Liell et al (2009) em Unidades Básicas de Saúde, onde a MP $=2,62$. Um estudo realizado em todas as regiões do Brasil encontrou como MP nacional 2,3 (OMS, 2005). O valor encontrado no nosso estudo foi superior ao indicado como racional pela OMS de até dois medicamentos por prescrição na atenção de saúde básica (OMS, 2005).

A taxa de prescrição de antibióticos foi maior do que em outros locais, como em Passo Fundo, 18,1\% (LIELL et al, 2009), e
Blumenau, 12,5\% (COLOMBO et al, 2004). Estes resultados superiores obtidos nos estudos citados podem estar relacionados ao período de coleta de dados corresponder aos meses de agosto/novembro e dezembro respectivamente, além disso, os estudos citados foram realizados em Unidades Básicas de Saúde. O valor observado de prescrições contendo antimicrobianos se encontra acima do limite de $20 \%$, sugerido como razoável pela OMS, e da taxa do país de 40,1\% (OMS, 2005). Este fato pode estar relacionado com o período em que as prescrições foram avaliadas ser de inverno no Rio Grande do Sul, no qual as infecções de trato respiratório superior são mais comuns, justificando o maior consumo de antibióticos. Desta forma, a elevada indicação de antimicrobianos, observada neste estudo, pode estar relacionada a fatores climáticos do período de coleta de dados, pois a sazonalidade é importante no comportamento epidemiológico de doenças infecciosas. Em um estudo com objetivo de identificar nas prescrições dispensadas em uma Unidade Básica de Saúde (UBS) do município de Ijuí/RS o perfil de utilização de antibióticos nos meses de verão e de inverno, foram avaliadas 10765 prescrições, através das quais verificaram que o período correspondente ao inverno (julho e agosto) foi responsável pela maior prescrição de antimicrobianos $(60,77 \%)$, caracterizando assim, uma variação sazonal na dispensação 
de antibióticos no local do estudo (WEBER et al.; 2010).

O principal problema quanto aos antibióticos é de que seu uso indiscriminado pode resultar em resistência de bactérias, aumentando os casos de infecções e, consequentemente, maior ônus para o sistema público de saúde e o usuário, gerando dificuldades para o controle da doença (PORTELLA et al.; 2012).

O número de medicamentos injetáveis foi inferior ao encontrado por Naves no Distrito Federal, 7,5\% (NAVES, SILVER, 2005); e em Passo Fundo, 14,3\% (LIELL et al, 2009); e da taxa nacional, 7,9\% (OMS, 2005). Este fato se deve as prescrições avaliadas serem do Pronto atendimento, sendo que os medicamentos injetáveis são aplicados no momento da consulta, não sendo necessária a prescrição para a dispensação na farmácia. Considerando este fato pode-se concluir que os valores de nossa pesquisa podem estar subnotificados em consequência da aplicação no local e cujos dados não se obteve acesso. Embora as prescrições de medicamentos injetáveis sejam importantes em situações, tais como a terapêutica de urgência, ou na absorção da substância em sua forma ativa, também podem ocorrer sérias consequências se forem erroneamente prescritos ou aplicados. Situações tais como, reações anafiláticas, necroses teciduais ou infecções por deficiência de assepsia devem ser cuidadosamente avaliadas. Essa via de aplicação é ainda, particularmente, sujeita a características culturais da sociedade, considerando a atitude da população frente a ela e o quanto essa postura pode influenciar os padrões de prescrição (SANTOS \& NITRINI; 2004).

Entre as prescrições avaliadas neste estudo, apenas $63 \%$ dos medicamentos prescritos seguiram a Relação Municipal de Medicamentos Essenciais (REMUME), sendo que a OMS recomenda que $100 \%$ das prescrições sejam realizadas a partir da lista de medicamentos essenciais (OMS, 2005). Esse número foi inferior ao encontrado no Distrito Federal, 85,3\% (COLOMBO et al, 2004), e em Passo Fundo, 77,3\% (LIELL et al, 2009), bem como menor que a média do país, no qual a porcentagem de medicamentos prescritos pertencentes à Lista de Medicamentos Essenciais foi de 78,3\% (OMS, 2005). A implantação de listas de medicamentos essenciais contribui para melhoria da qualidade da atenção à saúde, bem como para a eficácia da gestão dos medicamentos e prescrições médicas com mais qualidade. É importante apontar a necessidade de esta lista ser atualizada periodicamente, para melhor atender o perfil epidemiológico da população local (AZIZ et al.; 2012) além de verificar os motivos que levam os prescritores a não segui-la.

Quanto aos grupos de medicamentos mais prescritos de acordo com a classificação ATC, as classes terapêuticas mais prevalentes 
foram analgésicos, antibacterianos de uso sistêmico e antiinflamatórios e antireumáticos, resultado semelhante ao obtido por COLOMBO et al. (2004) avaliando o padrão de prescrição de medicamentos nas unidades de programa de saúde da família de Blumenau, onde $14,3 \%$ dos medicamentos prescritos foram analgésicos, seguido dos antibacterianos sistêmicos (12,5\%) e antiinflamatórios $(12,5 \%)$. FARIAS et al. (2007) verificou que as classes terapêuticas mais prevalentes foram antibacterianos de uso sistêmico $(21,0 \%)$, antiparasitários $(18,4 \%)$ e analgésicos e antipiréticos (15,4\%). Este resultado difere do encontrado neste estudo, pois o mesmo foi realizado em Unidades Básicas de Saúde da Família durante os meses de novembro e dezembro.

Não foi informado aos prescritores que estava-se avaliando as prescrições, para evitar que os mesmos, ao saberem da pesquisa, tendessem a fazê-las diferente do habitual. O fato das prescrições terem sido avaliadas durante apenas 1 mês do ano pode ter interferido nos resultados, visto que a ocorrência de determinadas doenças pode mudar com o decorrer do ano. Principalmente pelo fato do estudo ter sido desenvolvido durante meses de inverno no qual as infecções de trato respiratório são mais frequentes (OBRELI NETO et al., 2011).

\section{CONCLUSÃO}

Em relação aos indicadores do uso racional de medicamentos, as prescrições do pronto atendimento foram inadequadas na maioria dos indicadores avaliados. Pode-se observar que a maioria das prescrições continham medicamentos da REMUME. O número de antimicrobianos foi alto quando comparado a média nacional, provavelmente em decorrência da estação do ano mais susceptível a infecções do trato respiratório. Os medicamentos injetáveis foi menor em relação a outros estudos em outros locais do país. O número médio de medicamentos por receita foi maior do que o sugerido pela OMS, o que pode estar relacionado ao pronto atendimento ser destinado a emergências.

Essa observação, assim como os outros dados, pode direcionar esforços gerenciais e educativos no sentido da promoção do uso racional de medicamentos, e da formação profissional, pela busca de se atingir resultados mais próximos daqueles preconizados pelos órgãos internacionais, refletindo na qualidade da terapêutica empregada.

\section{REFERENCIAS}

ALBUQUERQUE, Verônica Maria Tavares de,. TAVARES, Cybelle Alves,. Avaliação de Indicadores de Medicamentos: Importância para a qualidade na prescrição médica. $\mathbf{R}$.

Bras. Farm. Hosp. Serv. Saúde. v.2 n.3, p. 31 -5 set./dez. 2011. 
AZIZ, M. M.; CALVO, M.C.M.; D'ORSI, E. Medicamentos prescritos aos idosos em uma capital do Sul do Brasil e a Relação Municipal de Medicamentos. Cad. Saúde Pública, v. 28, n. 1, 2012.

BRASIL, Ministério da Saúde. Conselho Nacional de Saúde. Resolução no 196, de 10 de outubro de 1996. Aprova as seguintes diretrizes e normas regulamentadoras de pesquisas envolvendo seres humanos. Comissão Nacional de Ética em Pesquisa. Brasília, 1996a. Disponível em: <http://www.ufrgs.br/bioetica/res 19696.htm\# conep>. Acesso em: 05 de julho de 2011.

BRASIL. Agência Nacional de Vigilância Sanitária (Brasil) Portaria no 1.179 , de 17 de junho de 1996. Dispõe sobre a descrição da Portaria. D.O.U - Diário Oficial da União; Poder executivo. Brasília. 18. Fev. 1996b.

BRASIL. MS. Portaria no 3.916, 30 out. 1998. Aprovar a Política Nacional de Medicamentos. Brasília, 1998. Disponível em: <http://www.saude.gov.br>. Acesso em: 1 jul. 2012.

CARVALHO, M.F.C.; LIEBER, N. S. R.; MENDES, G. B.; SECOLI, S. R.; RIBEIRO, E.; LEBRÃO, M. L.; DUARTE, Y. A. O. Polifarmácia entre idosos do Município de São Paulo - Estudo SABE. Rev Bras

Epidemiol. n.15, v.4, p. 817-827, 2012.

COLOMBO, D, et al. Padrão de Prescrição de Medicamentos nas Unidades de Programa de Saúde da Família de Blumenau. Rev. Bras. Cienc. Farm. n 40, v.4, 2004.

EV, L. S., GUIMARÃES, A. G., CASTRO, V. S. Avaliação das Prescrições Dispensadas em uma Unidade Básica de Saúde do Município de Ouro Preto, Minas Gerais, Brasil. Latin American Journal of Pharmacy - n.27, v.4, 2008.

FARIAS, A. D., CARDOSO, M. A. A., MEDEIROS, A.C.D., BELÉM, L.F., SIMÕES, M. O. S. Indicadores de prescrição médica nas unidades básicas de Saúde da
Família no município de Campina Grande, PB. Rev. bras. epidemiol., v. 10, n. 2, 2007.

FROHLICH, Samanta Etges; MENGUE, Sotero Serrate. Os indicadores de qualidade da prescrição de medicamentos da Organização Mundial da Saúde ainda são válidos?. Ciênc. saúde coletiva, v. 16, n. 4, 2011.

GIROTTO, E., SILVA, P.V. A prescrição de medicamentos em um município do Norte do Paraná. Rev Bras Epidemiol, n.9, v.2, p. 226-34, 2006.

LIELL, Andressa Pilonetto, et al. Indicadores do uso racional de medicamentos em ambulatório-escola. Revista da AMRIGS, n.53, v.4, p. 341-344, out.-dez, 2009. MARIN Nelly, et al. Assistência farmacêutica para gerentes municipais. Rio de Janeiro: Organização Pan-Americana da Saúde; 2003.

NAVES, J.O.S, SILVER, L.D. Avaliação da assistência farmacêutica na atenção primária no Distrito Federal. Rev. Saúde Pública. n. 39, v. 2, p. 223-230, 2005.

NETO, P. R. O., VIEIRA, J. C., CUMAN, R. K. N.. Impacto da atenção farmacêutica no uso racional de antimicrobianos em uma unidade básica de saúde no interior do Estado de São Paulo. Acta Scientiarum. Health Sciences, v. 33, n. 2, p. 159-164, 2011.

OBRELI NETO, P.R.; VIEIRA, J.C.; CUMAN, R.K.N. Impacto da atenção farmacêutica no uso racional de antimicrobianos em uma unidade básica de saúde no interior do Estado de São Paulo. Acta Scientiarum. Health Sciences, 33, n. 2, p. 159-64, 2011.

\section{OPAS/OMS. Avaliação da Assistência} Farmacêutica no Brasil / Organização PanAmericana da Saúde, Organização Mundial da Saúde; Ministério da Saúde - Brasília: Organização Pan-Americana da Saúde; BRASIL. Ministério da Saúde, 2005. 
PORTELA, A.S., SILVA, P.C.D., SIMÕES, M.O.S., MEDEIROS, A.C.D.,

MONTENEGRO, N.A.N. Indicadores de

prescrição e de cuidado ao paciente na

atenção básica do município de Esperança,

Paraíba, 2007. Epidemiol. Serv. Saúde, v.

21, n. 2, 2012.

SANTOS, V., NITRINI, S.M.O.O.

Indicadores do uso de medicamentos e de assistência ao paciente de serviços de saúde.

Rev. Saúde Pública. n.38, v. 6, p. 819-36, 2004.

SILVÉRIO, M.S.; LEITE, I.C.G. Qualidade das prescrições em município de Minas Gerais: uma abordagem farmacoepidemimiológica. Rev Assoc Med Bras. Juiz de Fora, n.56, v.6, p.675-680, 2010.

WEBER, B.R.; SCHERER, V.C.; OLIVEIRA, K.R.; COLET, C.F. Análise da variação sazonal da prescrição de antibióticos aos usuários de uma unidade básica de saúde do munícipio de Ijuí/RS. Revista Contexto \&

Saúde. Ijuí, v. 10, n. 19, Jul./Dez. 2010

World Health Organization [WHO].

Collaborating Centre for Drug Statistics

Methodologhy Guideline for ATC

classification and DDD assignment 3rd ed. Oslo: WHO Collaborating Centre for Drug Statistics Methodology; 2000. 268p. 